active index (DAI), Colon macroscopic damage index (CMDI), Tissue damage index (TDI) scores, weights, colon length and histologic manifestation were evaluated. Ratio of $\mathrm{T}$ helper cell type 17 (Th17)/regulatory T cells (Treg), expressions of differentiation-associated transducer and cytokines were detected. Relationship between Th17/Treg and peroxisome proliferatoractivated receptor-r (PPAR-r)/adenosine monophosphate-activated protein kinase (AMPK)/acetyl CoA carboxylase (ACC) signal pathways were analyzed.

Results Dietary n-3PUFA attenuated CD rats by reducing DAI, CMDI, TDI scores and colon shortening, promoted weight gain, and ameliorated histologic manifestations. N-3PUFA shifted Th17 into Treg by activating PPAR-r and downstream AMPK, while inhibiting ACC. Effect of restoring imbalance of Th17/Treg could be enhanced by PPAR-r agonist rosiglitazone, AMPK agonist AICAR and ACC antagonist TOFA, inhibited by PPAR-r antagonist GW9662, AMPK antagonist compound $\mathrm{C}$ and ACC agonist citric acid.

Conclusions Dietary n-3PUFA effectively protected and ameliorated $\mathrm{CD}$. The underlying mechanisms involved the restoration of Th17/Treg imbalance by regulating PPAR-r/AMPK/ACC signal pathways. (Figure 1).

\section{IDDF2020-ABS-0158 BRAF MUTATION INDUCES RAPID NEOPLASTIC TRANSFORMATION IN THE AGED AND EXTENSIVELY HYPERMETHYLATED INTESTINAL EPITHELIUM}

'Lochlan Fennell*, 'Alexandra Kane, 'Cheng Liu, 'Diane McKeone, 'Gunter Hartel, ${ }^{1}$ Catherine Bond, ${ }^{2}$ Mark Bettington, ${ }^{3}$ Barbara Leggett, ${ }^{1}$ Vicki Whitehall. ${ }^{1}$ QIMR Berghofer Medical Research Institute, Australia; ${ }^{2}$ Envoi Specialist Pathology, Australia; ${ }^{3}$ The Royal Brisbane and Women's Hospital, Australia

\subsection{6/gutjnl-2020-IDDF.28}

Background Sessile serrated lesions (SSL) are common in both young and old individuals, but the BRAF mutant cancers arising occur predominantly the elderly. DNA Methylation is uncommon in SSL from young patients. Here we interrogate the role of aging and DNA methylation in SSL initiation and progression.

Methods We used an inducible model of Braf mutation to direct recombination of the oncogenic Braf V637E allele to the murine intestine. BRAF mutation was activated after periods of aging, and histological and DNA methylation analysis was performed thereafter. We investigated DNA methylation alterations in human SSLs.

Results Inducing Braf mutation in aged mice was associated with a 10-fold relative risk of serrated lesions compared with young mice. Methylation analysis revealed extensive differences in age-associated DNA methylation between animals induced at 9 months versus wean; with relatively little differential Braf-specific methylation, implicating age-associated DNA methylation rather than Braf-specific DNA methylation in the heightened risk. DNA methylation at WNT pathway genes scales with age and Braf mutation accelerated age-associated DNA methylation. In human SSLs, increased epigenetic age was associated with high-risk serrated colorectal neoplasia.

Conclusions SSLs arising in the aged intestine are at a significantly higher risk of spontaneous neoplastic progression. These findings support a new conceptual model for serrated neoplasia whereby the risk of progression is related to the milieu of epigenetic alterations in the intestinal epithelium at the time of BRAF mutation, rather than the length of time since polyp initiation. This has implications for surveillance and chemopreventive strategies targeting the epigenome.

\section{IDDF2020-ABS-0174 ONSET OF HYPERTRIGLYCERIDEMIA IN RELATION TO DIETARY INTAKE, GUT MICROBIOME AND METABOLOMICS SIGNATURES AMONG HOME DWELLING ELDERLY}

${ }^{1}$ Hajar Fauzan Ahmad*, ${ }^{2}$ Josue Leonardo Castro Mejia, ${ }^{2}$ Lukasz Krych, ${ }^{2}$ Bekzod Khakimo ${ }^{3}$ Witold Kot, ${ }^{4}$ Rasmus Leidesdorff Bechshøft, ${ }^{4}$ Søren Reitelseder, ${ }^{2}$ Søren Balling Engelsen, ${ }^{5}$ Lars Holm, ${ }^{6}$ Karoline Faust. ${ }^{1}$ Faculty of Industrial Sciences and Technology, Department of Industrial Biotechnology, Universiti Malaysia Pahang, Malaysia; ${ }^{2}$ Department of Food Science, Faculty of Science, University of Copenhagen, Denmark; ${ }^{3}$ Department of Environmental Science, Aarhus University, Denmark; ${ }^{4}$ Institute of Sports Medicine Copenhagen, Department of Orthopedic Surgery M, Bispebjerg Hospital, Denmark; ${ }^{5}$ School of Sport, Exercise and Rehabilitation Sciences, University of Birmingham, UK; ${ }^{6}$ Department of Microbiology and Immunology, Rega Institute, KU Leuven, Belgium

\subsection{6/gutjnl-2020-IDDF.29}

Background The human gut is home for plethora of microbes including prokaryotic, eukaryotic and other microorganisms. During ageing, imbalances in the gut microbiota are associated with significant phenotypic effects for the host such as the development of metabolic disorders like changes in serum lipids levels, including general physiological decline. However, the presence of fungal communities and their possible association with host health are poorly understood. Therefore, we aim to elucidate trajectory for the progression of atherogenic dyslipidemia during ageing.

Methods The interplay between dietary intake, gut microbiota composition, plasma and fecal metabolome and clinical measurements were investigated. The gut bacterial and fungal compositions were determined by high-throughput sequencing of $\mathrm{V} 3$ region of $16 \mathrm{~S}$ rRNA and internal transcribed spacer (ITS2) gene amplicons, respectively. The plasma and fecal metabolomes were determined by GC-TOF-MS. Finally, the dietary intake records and the anthropometric/body-composition measurements at baseline were taken from 75 senior citizens aged 65 years old and above $(69.57 \pm 3.64)$.

Results At phyla, the gut is home to three main eukaryotic, namely Ascomycota, Basidiomycota and Zygomycota, with genera Penicillium, Candida, and Aspergillus being particularly common. Hypertriglyceridemia group (HG) was associated with low species richness as compared to Normotryglyceridemia group (NG), indicate by $\alpha$-diversity - Observed species, PD whole tree and Chao1 indices; $p<0.05$, and Bray-Curtis dissimilarity matrix-based analysis showed significant $(p<0.05)$ clustering according to fasting levels of circulating plasma triglycerides (Tg). Inversely, the hypertriglyceridemia clustering based on the prokaryotic component was not observed among both groups. Higher levels of $\mathrm{Tg}$ significantly associates with increased relative abundance of genus Penicillium, possibly mediated by a higher dietary fat intake (ANOVA, $p<0.05$ ), and Aspergillus and Guehomyces were positively associated with short-chain fatty acids (SCFAs) groups.

Conclusions Collectively, these findings suggest that the gut mycobiome dysbiosis is associated with hypertriglyceridemia, a known risk factor for the development of cardiovascular disease among the elders. 